\title{
Patrones Epidemiológicos del Trauma Dentoalveolar (Patología GES) en Pacientes Adultos Atendidos en un Centro de Trauma de Chile Durante 2 Períodos
}

\author{
Epidemiological Patterns of Traumatic Dental Injuries (GES Disease) in Adult \\ Patients Treated at a Chilean National Trauma Center during 2 Periods
}

\begin{abstract}
Francisca Velásquez,",; Claudia Mancilla*,; Ana Yelitza Niño*; Víctor Tirreau*; Juan Cortés-Araya*; María Cecilia Rojas $^{\star *}$; Enrico Escobar ${ }^{*, * *}$; Daniel Reyes-Court ${ }^{*, * *}$; Sergio Calleja*; Carolina Ulloa-Marín ${ }^{*}$ \&siao Hsin Sung-Hsieh ${ }^{*, * * *}$
\end{abstract}

VELÁSQUEZ, F.; MANCILLA, C.; NIÑO, A. Y.; TIRREAU, V.; CORTÉS-ARAYA, J.; ROJAS, M. C.; ESCOBAR, E.; REYES-COURT, D.; CALLEJA, S.; ULLOA-MARÍN, C. \& SUNG-HSIEH, H. H. Patrones epidemiológicos del trauma dentoalveolar (Patología GES) en pacientes adultos atendidos en un Centro de Trauma de Chile durante 2 períodos. Int. J. Odontostomat., 8(2):191-199, 2014.

RESUMEN: El trauma dentoalveolar (TDA) constituye un conjunto de lesiones que comprometen los dientes o a sus estructuras periodontales. En Chile, desde el año 2007, la primera consulta/tratamiento de urgencia del TDA está cubierta por la Ley N $N^{\circ}$ 19.966, para todas las personas afiliadas al Fondo Nacional de Salud (FONASA) y a las Instituciones de Salud Previsional Privadas (ISAPRE) a través del Programa de Garantías Explícitas en Salud (GES). Escasos estudios nacionales se han realizado en TDA de adultos y ninguno en relación al impacto del GES en estas lesiones. Se realizó un estudio retrospectivo, descriptivo y transversal de TDA con los Datos de Urgencia de todos los pacientes adultos atendidos en el Hospital de Urgencia Asistencia Pública. Se compararon las variables clínicas, etiológicas, demográficas y sociales entre 2 períodos: pre-GES (1 de Julio de 2005 al 30 de Junio de 2006) y post-GES (1 de julio de 2012 al 31 junio de 2013). En los 2 períodos se observó una mayor frecuencia de TDA en el sexo masculino del grupo de 2029 años, producidos en la mayoría de los casos por violencia interpersonal. Sin embargo se observó en el período postGES una mayor consulta de TDA por Accidente vehículo-motorizado, presentándose lesiones de mayor gravedad. A pesar de la implementación del GES, se observó una alta frecuencia de TDA no tratados, esto podría deberse a la gravedad del estado sistémico del paciente (postergando el tratamiento de TDA), a la falta de insumos o a la inequidad en la entrega de recursos a los servicios de salud. Es necesario realizar más estudios y vigilancia de parte de la autoridad sanitaria para mejorar las garantías del GES en el tratamiento de los TDA.

PALABRAS CLAVE: trauma dentoalveolar, epidemiología, adultos.

\section{INTRODUCCIÓN}

El trauma dentoalveolar (TDA) constituye un conjunto de lesiones que comprometen los dientes o sus estructuras de soporte, como consecuencia de un impacto violento directo o indirecto (Andreasen et al., 2009). La presentación clínica de estas lesiones depende de la cantidad de energía del impacto, la forma y dirección del objeto que causa el trauma y además de la resiliencia de las diversas estructuras bucales. Esto combinado con la edad del paciente y los antecedentes mórbidos, genera una amplia posibilidad de lesiones traumáticas (Acevedo et al., 2006).
De acuerdo a la literatura, el TDA es una patología que afecta a individuos de todas las edades, con mayor prevalencia en niños. Se postula que esta mayor prevalencia se debe a factores anatómicos como una dentición más expuesta a ser traumatizada, mayor actividad física con conductas más riesgosas (Onetto et al., 1994) y a la falta de coordinación debido a sistemas sensoriales menos desarrollados, impidiéndoles a realizar maniobras reflejas de protección como girar la cabeza hacia un lado al momento de caer (Shaikh \& Worrall, 2002).

\footnotetext{
Departamento de Cirugía y Traumatología Bucal y Maxilofacial, Facultad de Odontología, Universidad de Chile, Santiago, Chile.

** Hospital de Urgencia Asistencia Pública (HUAP), Santiago, Chile.

*** Oral and Maxillofacial Surgery Department, University of Michigan, Ann Arbor, Michigan, EEUU.

Financiado parcialmente por el proyecto FONIS SA11I2082 (HHSH, CUM)
} 
VELÁSQUEZ, F.; MANCILLA, C.; NIÑO, A. Y.; TIRREAU, V.; CORTÉS-ARAYA, J.; ROJAS, M. C.; ESCOBAR, E.; REYES-COURT, D.; CALLEJA, S.; ULLOA-MARÍN, C. \& SUNG-HSIEH, H. H. Patrones epidemiológicos del trauma dentoalveolar (Patología GES) en pacientes adultos atendidos en un Centro de Trauma de Chile durante 2 períodos. Int. J. Odontostomat, 8(2):191-199, 2014.

En Chile, desde el año 2007, el tratamiento de urgencia del TDA está cubierto por la Ley $\mathrm{N}^{\circ}$ 19.966 Decreto $\mathrm{N}^{\circ} 44$, para todas las personas afiliadas al Fondo Nacional de Salud (FONASA) e Instituciones de Salud Previsional (ISAPRES), a través del Programa de Garantías Explícitas en Salud (GES). Es decir, el acceso, calidad, oportunidad y protección financiera del tratamiento de urgencia de esta patología está asegurado por esta Ley. Uno de los principales problemas del TDA es su alto costo, tanto para la sociedad como para el individuo y este costo se hace mayor si el TDA no recibe un tratamiento de urgencia de manera oportuna y adecuada, lo que puede producir secuelas funcionales, psicológicas y estéticas a los pacientes (Caldas \& Burgos, 2001; Santos et al., 2010). La cobertura universal del GES para el TDA ha sido un gran avance para mejorar la salud oral de la población chilena. Sin embargo este beneficio es sólo para la primera consulta de urgencia, dejando el costo de los tratamientos rehabilitadores o definitivos como responsabilidad individual de cada paciente afectado.

De acuerdo a la literatura, los patrones epidemiológicos de los TDA están ligados a factores sociales, culturales y ambientales (Glendor, 2009). La tendencia de una vida moderna más ajetreada, con mayor práctica de deportes de contacto, aumento de la violencia en la ciudad de Santiago (Goldstein et al., 2003), incremento de la expectativa de vida de la población y aumento de un $49 \%$ del parque automotor en el país (de 2.657.892 en 2006 a 3.973.913 en 2012) (I.N.E.), podría predisponer a un aumento de la frecuencia de los TDA en la población adulta chilena. A pesar de ser una patología GES, la epidemiología de TDA en la población adulta chilena ha sido poco estudiada y ninguno de estos estudios se ha enfocado en el impacto de la implementación del GES sobre el comportamiento de estas lesiones.

El Hospital de Urgencia Asistencia Publica (HUAP), es un Centro de Referencia de Trauma a nivel Nacional para pacientes adultos (individuos mayores de 15 años), y uno de los 3 Hospitales estatales de Santiago que cuenta con un Servicio de Urgencia Dental con un sistema de atención continuada de 24 horas durante los 7 días de la semana (24/7), y recibe habitualmente pacientes afectados por trauma dentoalveolar. El objetivo de este estudio es describir el perfil epidemiológico de TDA en la población adulta pre y post implementación del GES.

\section{MATERIAL Y MÉTODO}

Estudio retrospectivo, descriptivo y transversal, en el cual la recopilación de los datos se realizó en el HUAP y se llevó a cabo en 2 períodos. En el año 2009 con la autorización de la Dirección del Hospital, se revisaron los datos de atención de urgencia (DAU) de un período Pre-GES (1 de Julio de 2005 al 30 de Junio de 2006). En el año 2013 con la autorización del Director del Hospital y la aprobación del Comité Ético-Científico del Servicio de Salud Metropolitano Central (bajo la regulación de las Leyes $N^{\circ} 20.584$ y No $20.120)$ se revisaron los DAU de un período post-GES (1 de julio de 2012 al 31 junio de 2013).

EI Universo estuvo constituido por los DAU de todos los pacientes que consultaron al HUAP, ya sea por urgencia médica u odontológica. La muestra correspondió a los DAU de todos los pacientes diagnosticados con TDA. El muestreo correspondió al tipo no probabilístico por conveniencia.

Las variables a estudiar se agruparon en cinco categorías:

1. Variables clínicas: Se consideró como TDA a toda lesión traumática que afecte al diente propiamente tal, y a sus estructuras de soporte, consecutiva a un impacto violento. Sin embargo, respecto del tejido afectado hay diagnósticos específicos de TDA, descritos en la Guía Clínica de Urgencias Odontológicas del Ministerio de Salud del año 2011 (MINSAL, 2011).

- Lesiones a los tejidos dentarios: fracturas coronarias (no complicada, complicada), fractura corono-radicular, fractura radicular y fractura alveolar.

- Lesiones a los tejidos de soporte: concusión, subluxación, luxación (extrusiva, lateral, intrusiva) y avulsión.

2) Variable etiológica: corresponde a la causa por la cual se produjo el TDA, tales como caída, violencia interpersonal (asalto, agresión), accidente en vehículo motorizado, violencia intrafamiliar, actividades deportivas y otros.

3) Variables demográficas: edad (en años), sexo (masculino o femenino).

4) Variables sociales: ingesta de bebidas alcohólicas. 
VELÁSQUEZ, F.; MANCILLA, C.; NIÑO, A. Y.; TIRREAU, V.; CORTÉS-ARAYA, J.; ROJAS, M. C.; ESCOBAR, E.; REYES-COURT, D.; CALLEJA, S.; ULLOA-MARÍN, C. \& SUNG-HSIEH, H. H. Patrones epidemiológicos del trauma dentoalveolar (Patología GES) en pacientes adultos atendidos en un Centro de Trauma de Chile durante 2 períodos. Int. J. Odontostomat., 8(2):191-199, 2014

5) Variable manejo de urgencia: tipo de tratamiento recibido.

Para el registro de los datos recolectados de los pacientes en el período 2005-2006 se utilizó el programa Microsoft office Access (versión 2007, USA). Para el período 2012-2013 se confeccionó un registro de datos (CRF) que considera las variables anteriormente mencionadas, siendo los datos registrados traspasados posteriormente al programa Epilnfo (versión 5.3, CDC, USA). En ambos períodos fueron omitidos los datos personales (los relativos a cualquier información concerniente a personas naturales, identificadas o identificables) de los pacientes. Los registros fueron realizados en las dependencias del HUAP y la información obtenida fue registrada y almacenada de forma anonimizada y confidencial, con un carácter netamente estadístico.

Los resultados fueron analizados con estadística de tipo descriptivo. Se evaluó la distribución del TDA respecto de las variables descritas anteriormente.

\section{RESULTADOS}

En los períodos pre-GES (2005-2006) y postGES (2012-2013) se revisaron el DAU del total de los consultantes que fueron 130.414 y 146.392 , respectivamente (Tabla I).
Tabla I. Características demográficas y resumen de lesiones de períodos pre-GES y post-GES.

\begin{tabular}{lcc}
\hline & $\begin{array}{c}\text { Período } \\
\text { pre-GES } \\
\mathbf{( 2 0 0 5 - 2 0 0 6 )}\end{array}$ & $\begin{array}{c}\text { Período } \\
\text { post-GES } \\
\mathbf{( 2 0 1 2 - 2 0 1 3 )}\end{array}$ \\
\hline Total de consultas en HUAP & 130.414 & 146.932 \\
n Individuos con TDA M/F & $210 / 73$ & $162 / 66$ \\
Rango de edad & $15-87$ & $15-89$ \\
n de TDA & 341 & 443 \\
n LTB & 354 & 193 \\
\hline
\end{tabular}

HUAP = Hospital de Urgencia de Asistencia Pública, n= número, TDA $=$ Traumatismo dentoalveolar, $M=$ Masculino, $F=$ Femenino, LTB= lesión de tejido blando.

El rango de edad de los pacientes con TDA al momento del trauma fue entre los $15-87$ años (pre-GES) y 15-89 años (post-GES), con un promedio de 35 años. En ambos períodos los casos de TDA se producen con mayor frecuencia en los menores de 49 años, con un punto más alto en el grupo de 20-29 años, y disminuye su frecuencia con el aumento de la edad (Fig. 1).

Se observó un predominio del sexo masculino en la mayoría de los rangos etáreos, con una relación entre masculino y femenino de 2,9:1 (pre-GES) y 2,5:1 (post-GES) (Fig. 1).

En ambos períodos la mayoría de los pacientes están afiliados a FONASA. Se observó una mayor frecuencia de TDA en pacientes afiliados al tramo $A$ en el período pre-GES y al B en el post-GES (Fig. 2).

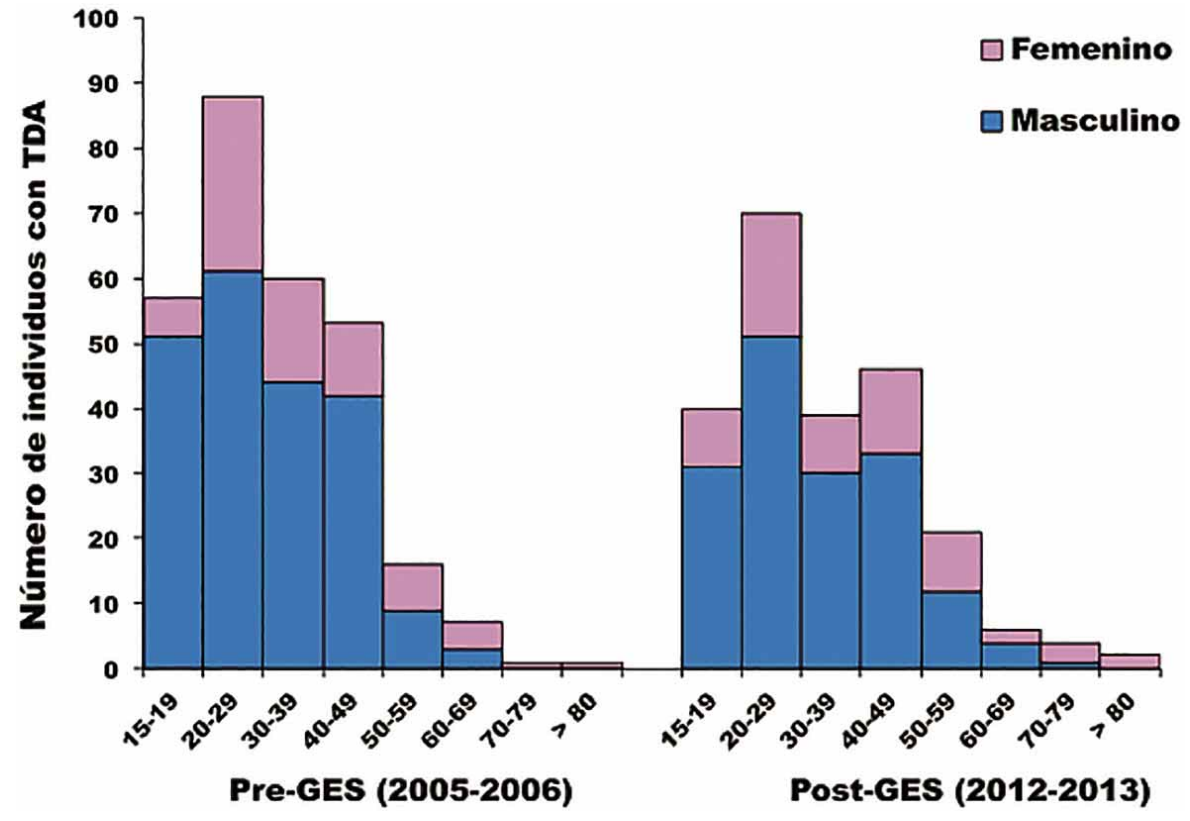

Fig. 1. Frecuencia de TDA según sexo y grupo etario en los 2 períodos. 
VELÁSQUEZ, F.; MANCILLA, C.; NIÑO, A. Y.; TIRREAU, V.; CORTÉS-ARAYA, J.; ROJAS, M. C.; ESCOBAR, E.; REYES-COURT, D.; CALLEJA, S.; ULLOA-MARÍN, C. \& SUNG-HSIEH, H. H. Patrones epidemiológicos del trauma dentoalveolar (Patología GES) en pacientes adultos atendidos en un Centro de Trauma de Chile durante 2 períodos. Int. J. Odontostomat., 8(2):191-199, 2014

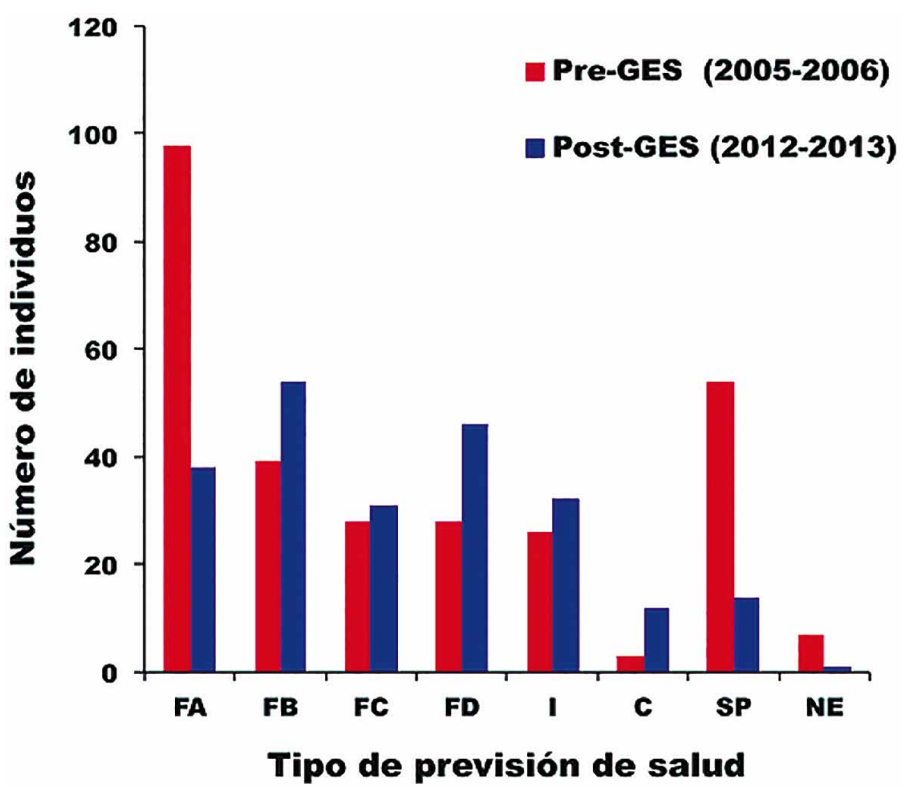

Fig. 2. Frecuencia de TDA según tipo de previsión de salud en los 2 períodos. $\mathrm{FA}=$ Fonasa $\mathrm{A}, \mathrm{FB}=$ Fonasa $\mathrm{B}, \mathrm{FC}=$ Fonasa $\mathrm{C}, \mathrm{FD}=$ Fonasa $\mathrm{D}, \mathrm{I}=$ Isapre, $\mathrm{C}=$ Convenios, $\mathrm{SP}=$ Sin previsión, $\mathrm{NE}=$ no especificado.

La causa más frecuente en ambos periodos fue la violencia interpersonal (VIP) con un $60,9 \%$ en el período pre-GES y $35,2 \%$ en el post-GES, seguido por las caídas y los accidentes en vehículo motorizado (AVM) (Fig. 3). Sólo $26(14,5 \%)$ pacientes del período pre-GES y $28(13,6 \%)$ del post-GES estuvieron bajo la influencia del alcohol al momento de la lesión. Las etiologías de TDA asociadas al consumo de alcohol fueron mayoritariamente por VIP. Durante el periodo post-GES, en el $30,6 \%$ de los TDA registrados no especificó el lugar del suceso. La vía pública fue, entre los lugares identificados, el más frecuente representando el $52,5 \%$ del total, seguido por el hogar con un $10,7 \%$, luego el lugar de trabajo (2,9\%), área deportiva $(1,9 \%)$ y lugar de estudio $(0,5 \%)$.

El tipo de lesión de tejido blando facial más frecuentemente observado con TDA en el período pre-GES fueron las contusiones $(65,8 \%)$, seguida por heridas $25,2 \%$ y erosiones $8,2 \%$; a diferencia de lo observado en post-GES que fueron heridas (laceración, transfixiante o avulsión) con un $46,1 \%$, contusiones $43,5 \%$ y erosiones $10,4 \%$.

En el período pre-GES se observó un máximo de 3 dientes con TDA por paciente, en cambio en el post-GES el número de dientes lesionados por paciente se incrementó a 6 (Fig. 4).

En el período pre-GES los 283 pacientes diagnosticados con TDA, presentaron un total de 341 lesiones, de éstas $188(55,1 \%)$ corresponden a lesiones en los tejidos duros o pulpa dentaria; $142(41,7 \%)$ a lesiones de los tejidos periodontales y $11(3,2 \%)$ a fracturas protésicas. Estos resultados son similares a los del período post-GES, de un total de 443 lesiones, $240(54,1 \%)$ fueron en tejidos duros $\mathrm{y} /$ o pulpa dentaria y $203(45,9 \%)$ en tejidos de soporte dentario, no encontrando registro de fracturas protésicas (Fig. 5). El tipo de lesión más frecuente en ambos períodos fue la Fractura coronaria (complicada y no complicada) seguida por lesión de los tejidos de soportes dentarios. En el período post-GES se observó que las Fracturas coronarias no complicadas $(22,3 \%)$ fueron las lesiones más frecuentes, seguidas por concusión $(14,4 \%)$ y subluxación $(14,4 \%)$ (Fig. 5).

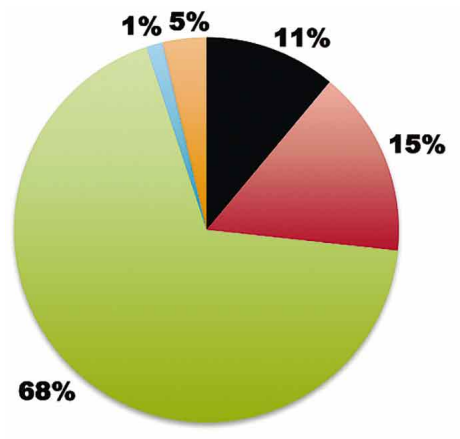

Pre-GES 2005-2006

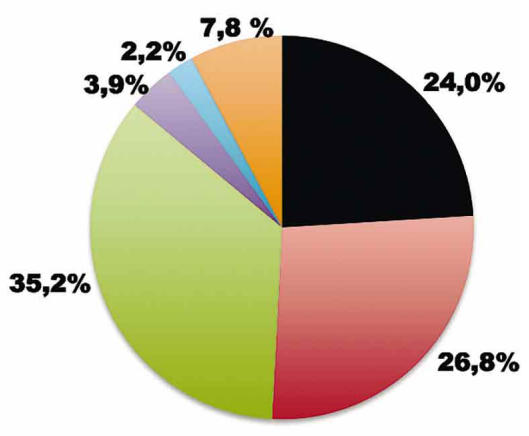

Post-GES 2012-2013

Fig. 3. Frecuencia de TDA (en porcentaje) según etiología de los 2 períodos. AVM=Accidente vehículo motorizado, $\mathrm{VIP}=$ violencia interpersonal, $\mathrm{VIF}=$ violencia intrafamiliar, $\mathrm{LD}=$ lesiones deportivas. 
VELÁSQUEZ, F.; MANCILLA, C.; NIÑO, A. Y.; TIRREAU, V.; CORTÉS-ARAYA, J.; ROJAS, M. C.; ESCOBAR, E.; REYES-COURT, D.; CALLEJA, S.; ULLOA-MARÍN, C. \& SUNG-HSIEH, H. H. Patrones epidemiológicos del trauma dentoalveolar (Patología GES) en pacientes adultos atendidos en un Centro de Trauma de Chile durante 2 períodos. Int. J. Odontostomat., 8(2):191-199, 2014.

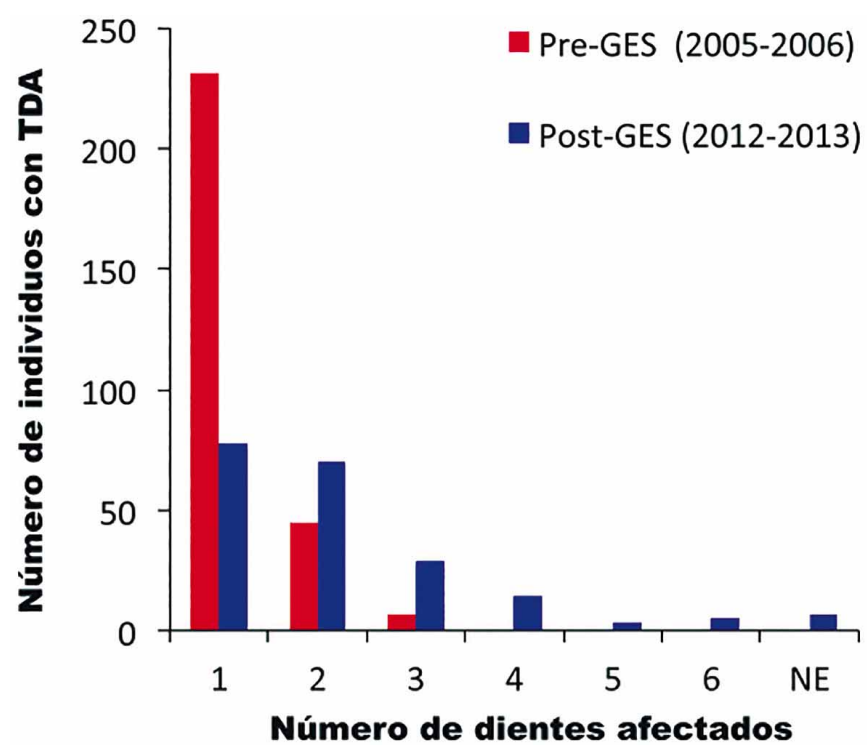

Fig. 4. Frecuencia de dientes traumatizados por individuos (pacientes).

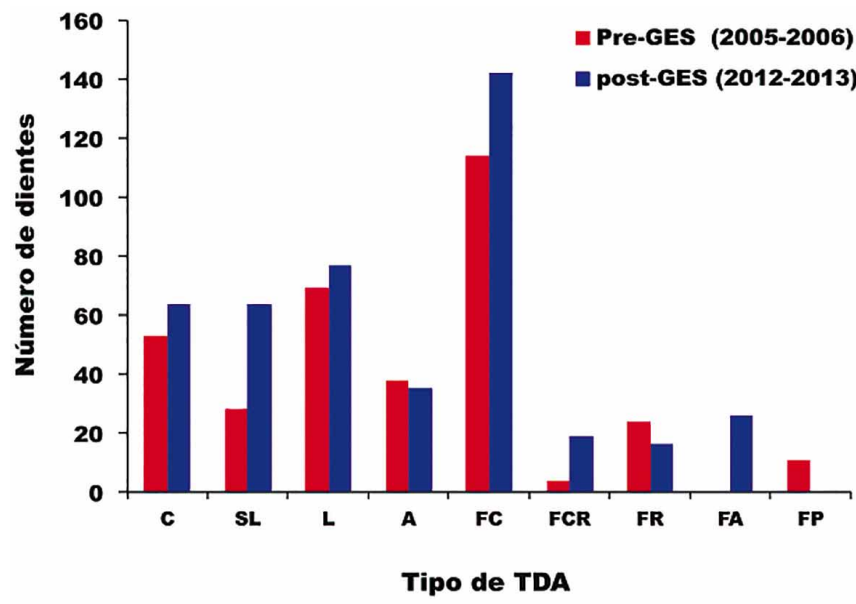

Fig. 5. Frecuencia de tipo de TDA. Clasificación de tipo de TDA basada en la guía de Tratamiento de Urgencia del MINSAL del 2011.

De acuerdo a la distribución de TDA según dientes se observó que los dientes anteriores maxilares, especialmente los dientes 1.1 y 2.1 fueron los más afectadas; quienes presentan mayoritariamente fractura coronaria no complicada, subluxación y luxación lateral (Fig. 6).

De las 443 lesiones diagnosticadas en el período post-GES, sólo $223(50,3 \%)$ recibieron tratamientos, las 220 lesiones restantes, sólo fueron examinadas, diagnosticadas y referidas. Dentro de los tratamientos más realizados: 51 dientes fueron extraídos, 49 lesiones recibieron reposicionamiento y ferulización, y 40 requirieron de férula flexible (Fig. 7).

\section{DISCUSIÓN}

Se observó una menor frecuencia de TDA en el período post-GES. Esta disminución de las consultas por TDA es razonable, considerando que por Ley, el Estado debe garantizar la cobertura de esta patología, lo que nos hace suponer que ha habido un incremento de Centros de Salud pertenecientes a la Red de prestadores GES disponibles para tratar estas lesiones. Por lo tanto, las personas afectadas acudirían a los Centros de Salud más cercanos a su domicilio o del lugar del suceso, en vez de recurrir al HUAP, que en época preGES era uno de los pocos centros de Santiago que otorgaba el tratamiento de urgencia de TDA.

La mayor frecuencia de TDA en los menores de 49 años observados en ambos períodos (Fig. 1), podría ser porque los grupos más afectados corresponden a la fuerza laboral del país, los cuales al ser activos resultan más expuestos a este tipo de lesiones. La menor frecuencia de TDA en los mayores de 60 años se puede deber a que llevan una vida más sedentaria o son desdentados (parcial o totalmente). Estudio en adultos chilenos (>60 años) observaron que el $50 \%$ de ellos son desdentados totales maxilares y $27,3 \%$ mandibulares (Urzúa et al., 2009). La proporción entre el sexo masculino y femenino de 2,9:1 (pre-GES) y 2,5:1 (post-GES) (Fig. 1) fue similar a la observada en otros estudios (Abdul Rahman et al., 2010; Acevedo et al.; Locker, 2007).

Se observó una mayor frecuencia de TDA en pacientes afiliados al tramo $A$ en el período pre-GES y al B en el post-GES (Fig. 2), siendo estos los usuarios regulares de los hospitales públicos, constituyen los grupos de personas de menores ingresos económicos que no requieren realizar copagos en las patologías GES. Interesante destacar que la cantidad de pacientes beneficiarios de ISAPREs no ha variado en los 2 períodos, considerando que sus garantías del tratamiento de urgencia del TDA están cubiertas por GES desde el 2007. Los pacientes ISAPRE que acuden a un centro perteneciente a la Red de prestadores de su ISAPRE sólo deben realizar un copago hasta un máximo de $20 \%$ del arancel referencial (\$21.850) para la primera consulta de urgencia de TDA, lo que corresponde a $\$ 4.370$, monto definitivamente menor a lo que tendrían que pagar en caso de acudir al HUAP o a algún 
VELÁSQUEZ, F.; MANCILLA, C.; NIÑO, A. Y.; TIRREAU, V.; CORTÉS-ARAYA, J.; ROJAS, M. C.; ESCOBAR, E.; REYES-COURT, D.; CALLEJA, S.; ULLOA-MARÍN, C. \& SUNG-HSIEH, H. H. Patrones epidemiológicos del trauma dentoalveolar (Patología GES) en pacientes adultos atendidos en un Centro de Trauma de Chile durante 2 períodos. Int. J. Odontostomat., 8(2):191-199, 2014

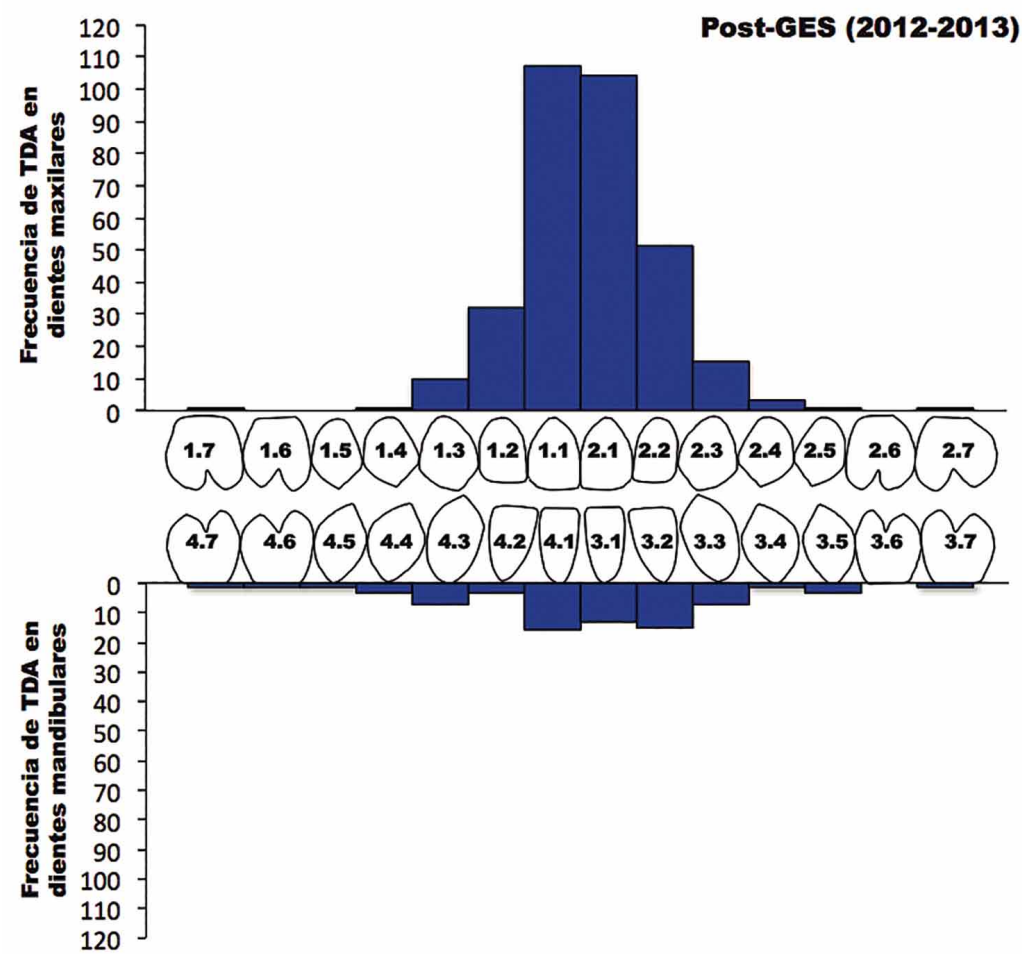

Fig. 6. Frecuencia de TDA según diente afectado. Se utilizó para la numeración de los dientes la nomenclatura de la Federación Dental Internacional (ISO 3950).

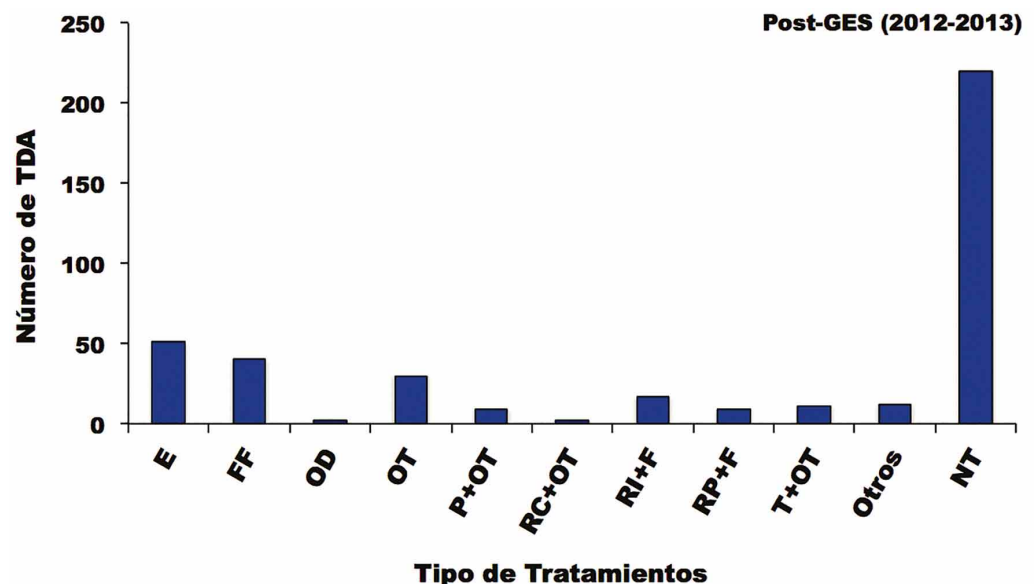

Fig. 7. Frecuencia de tratamientos recibidos por los pacientes con TDA. $\mathrm{E}=$ exodoncia, $\mathrm{FF}=$ férula flexible, $\mathrm{OD}=$ obturación definitiva, $\mathrm{OT}=$ obturación temporal, $\mathrm{P}=$ pulpotomía, $\mathrm{RC}=$ recubrimiento directo, $\mathrm{RI}=$ reimplante, $\mathrm{RP}=$ reposicionamiento, $\mathrm{F}=$ ferulización, $\mathrm{T}=$ trepanación, NT=no tratamiento.

otro Servicio de Salud estatal/municipalizado. La probable explicación del uso del Servicio de Salud Público por parte de los pacientes bajo el régimen de ISAPREs podría ser la falta de conocimiento de la cobertura GES o falta de Servicio de Atención de Urgencia Odontológica tipo 24/7 dentro de la red cerrada de prestadores designadas por la ISAPRE. Más estudios se requieren para confirmar o rechazar estas hipótesis en relación a los usuarios de ISAPREs.

La causa más frecuente en ambos periodos fue la VIP (Fig. 3). Estos resultados son similares al estudio nacional realizado en el año 2002, pero difieren de los obtenidos en estudios internacionales que describen AVM como la etiología principal, esta discrepancia probablemente se debe a que estos estudios fueron realizados en países cuyas leyes del tránsito son más flexibles y menos severas, además de deficientes programas de seguridad vial (Abdul Rahman et al.; Ramli et al., 2011). Es interesante destacar que AVM es la tercera causa más prevalente en ambos períodos, sin embargo su proporción con VIP en el período pre-GES es de 1:6 a diferencia del período de post-GES que es de 1:1,5. De acuerdo a la Comisión Nacional de Seguridad de Tránsito (CONASET), con la implementación del uso obligatorio de cinturón de seguridad, o también la aplicación de la Ley de Tolerancia Cero (Ley $N^{\circ} 20.580$, modifica Ley $\left.\mathrm{N}^{\circ} 18.290\right)$, la personas fallecidas en AVM disminuyeron de 1.652 en 2006 a 1.523 en 2012 . No obstante, los siniestros aumentaron en un $37 \%$ (44.839 en 2006 a 61.791 en 2012) al igual que los lesionados que aumentaron en un 13\% (47.025 en 2006 a 53.225 en 2012) (CONASET, 2012; SIEC, 2012). Por lo tanto, el incremento de casos de TDA por AVM observado en este estudio podría deberse a este aumento de siniestros y/ o al aumento de los Servicios de Atención Pública de Urgencia (SAPU), en que los pacientes con TDA secundario a VIP acuden a su centro de urgencia más cercano; y como HUAP es un Centro de Trauma de Referencia Nacional, probablemente recibiría pacientes de mayor gravedad como los del AVM. Más estudios a nivel nacional deben ser realizados para comprobar si estos patrones se reflejan en otros centros del país. 
VELÁSQUEZ, F.; MANCILLA, C.; NIÑO, A. Y.; TIRREAU, V.; CORTÉS-ARAYA, J.; ROJAS, M. C.; ESCOBAR, E.; REYES-COURT, D.; CALLEJA, S.; ULLOA-MARÍN, C. \& SUNG-HSIEH, H. H. Patrones epidemiológicos del trauma dentoalveolar (Patología GES) en pacientes adultos atendidos en un Centro de Trauma de Chile durante 2 períodos. Int. J. Odontostomat., 8(2):191-199, 2014

En nuestro país, el consumo excesivo de alcohol es uno de los principales problemas de salud que afecta a la población. Al año mueren 7.600 personas por causas asociadas a su ingesta; casi un millón de chilenos es "bebedor problema" y sus edades fluctúan principalmente entre los 15 y los 35 años (SENDA, 2012). Los siniestros de tránsito en los cuales se asoció como causa basal el consumo de alcohol en los conductores fueron 2.927 sucesos en el año 2006 y 3.130 en el año 2012 (Solimano, 2006; SIEC). Además, varios autores relatan que existe una fuerte asociación entre el consumo de alcohol y la VIP, ya que los bebedores usualmente participan en actividades peligrosas como peleas o agresiones. Esta relación fue observada por Laverick et al. (2008) en el Reino Unido, donde un $72 \%$ de los traumas faciales producidos por VIP estaban asociados a ingesta de alcohol. La menor frecuencia de casos de TDA con ingesta de alcohol, 14,5\% en el período pre-GES y $13,6 \%$ en el post-GES, observadas en el presente estudio puede deberse a problemas de registros. El ingreso en el DAU de la alcoholización del paciente se realiza generalmente cuando ésta es evidente o cuando el paciente ha sido sometido a una alcoholemia. Un registro sistemático de la presencia de alcohol en estos pacientes se transformaría en una información relevante a la hora de planificar medidas preventivas.

En ambos períodos se observó una mayor frecuencia de lesiones a los tejidos duros o pulpa dentaria, seguidas por lesiones a los tejidos periodontales. Estos resultados difieren con lo observado en otro estudio realizado en Santiago (año 2002) que encontraron una mayor frecuencia de TDA con compromiso de los tejidos periodontales $(56,42 \%$ ) (Acevedo et al.). El incremento de lesiones en los tejidos duros más el aumento de la cantidad de dientes con TDA por paciente (Fig. 4) y la mayor cantidad de lesiones tipo heridas (laceración, transfixiante o avulsión) observadas en el período post-GES nos hace sospechar de lesiones de mayor gravedad asociadas al TDA en este período. La explicación podría ser que al haber mayor cantidad de Centros de Salud para el tratamiento de los TDA, sólo aquellos con lesiones de mayor gravedad son derivados y atendidos en el HUAP.

Similar a lo descrito en la literatura (Acevedo et al.; Glendor, 2008; Guedes et al., 2010), en ambos períodos la fractura coronaria (complicada y no complicada) fue la lesión más frecuente, siendo los incisivos centrales los dientes más comprometidos. La situación de vulnerabilidad de estos dientes, que se pueden encontrar en protución o con cobertura labial inadecuada, podría explicar estos resultados. Es importante identificar el o los tipos de lesiones más frecuentes para que el Estado pueda ofrecer una canasta de GES que sea capaz de cubrir los gastos del tratamiento de urgencia, reservando los fondos de los Centros de Salud al resto de los pacientes portadores de patologías no cubiertas por GES.

El tratamiento de urgencia de las fracturas coronarias no complicadas es la obturación, que puede ser temporal o definitiva, sin embargo, sólo un $36 \%$ (32 de 89) de éstas recibieron el tratamientos. La baja frecuencia de TDA tratados sugieren lesiones que no recibieron el tratamiento adecuado según los protocolos del MINSAL, lo que podría traer mayores consecuencias para el paciente a medida que transcurre el tiempo y con ello, el incremento del costo final del tratamiento, independiente de quien sea el encargado de su financiamiento (Caldas \& Burgos; Santos et al.). La falta de oportunidad para brindar el tratamiento adecuado se podría deber a la gravedad del estado general del paciente, a la falta de insumos o a la inequidad en la entrega de recursos a los servicios de salud.

Las debilidades de este estudio estarían relacionadas con el registro de los datos obtenidos del DAU, tales como letras ilegibles $u$ omisión de datos (en un $21,3 \%$ no se especificó la etiología del trauma y en un $30,6 \%$ el lugar del suceso); y el desconocimiento de si los clínicos tratantes están calibrados en diagnósticos y tratamientos de TDA.

La Implementación del GES en el tratamiento de Urgencia de TDA aparentemente ha permitido que la población tenga un mejor acceso y oportunidad al tratamiento, ayudando a un descongestionamiento del HUAP para atención de otras patologías. Sin embargo, aún persisten importantes brechas relacionadas con las garantías de protección financiera y/o calidad de tratamientos. Esto puede deberse a escasez de insumos para llevar a cabo los tratamientos descritos en los protocolos o a la inadecuada previsión referida a la demanda esperada. Es necesario más estudios y vigilancia de parte de la autoridad sanitaria para mejorar las garantías del GES en el tratamiento de los TDA.

AGRADECIMIENTOS. Agradecemos la colaboración de la Sra. Pilar Barrera por su ayuda en secretaría; de Sra. Gloria Valenzuela con los datos estadísticos del HUAP; Sras. Paola Barrueto y Georgina Díaz por facilitarnos el registro de los datos; y a FONIS por el financiamiento. 
VELÁSQUEZ, F.; MANCILLA, C.; NIÑO, A. Y.; TIRREAU, V.; CORTÉS-ARAYA, J.; ROJAS, M. C.; ESCOBAR, E.; REYES-COURT, D.; CALLEJA, S.; ULLOA-MARÍN, C. \& SUNG-HSIEH, H. H. Patrones epidemiológicos del trauma dentoalveolar (Patología GES) en pacientes adultos atendidos en un Centro de Trauma de Chile durante 2 períodos. Int. J. Odontostomat., 8(2):191-199, 2014.

VELÁSQUEZ, F.; MANCILLA, C.; NIÑO, A. Y.; TIRREAU, V.; CORTÉS-ARAYA, J.; ROJAS, M. C.; ESCOBAR, E.; REYES-COURT, D.; CALLEJA, S.; ULLOA-MARÍN, C. \& SUNG-HSIEH, H. H. Epidemiological patterns of traumatic dental injuries (GES Disease) in adult patients treated at a Chilean National Trauma Center during 2 periods. Int. J. Odontostomat., 8(2):191-199, 2014.

ABSTRACT: Traumatic dental injury (TDI) is a group of injuries that affect hard dental tissues and/or periodontal structures. Since 2007 the first emergency treatment/consult of TDI, for both the National Health Fund (FONASA) and profit private insurer (ISAPRE) affiliates, is guaranteed in the Regulation of Explicit Health Guarantees (GES) established by Chilean Law 19.966. Few national TDI studies in adults have been carried out, and none in relation to the impact of GES in this type of lesion. A retrospective cross sectional study of emergency charts of all adult patients attended at the Hospital de Urgencia Asistencia Pública. Etiological, clinical, demographic and social variables were compared between 2 time periods, Pre-GES period (July, 12005 to June, 30 June 2006) versus Post-GES period (July, 12012 to June, 31 2013). A high incidence of TDI caused by interpersonal violence in males between 20 and 29 years old was observed in both periods. However, an increased TDI with more severe injuries caused by automobile accident was observed during the post-GES period. In spite of GES implementation, high frequency of non-treated TDI was seen in the present study, this could be due to the severity of the patient's systemic condition (delaying the TDI treatment), a lack of resources and/ or inequity in the delivery of these healthcare resources. More studies and surveillance programs by the Government are needed to improve TDI treatment guarantees, and as well as regular assessment of GES compliance.

KEY WORDS: traumatic dental injuries, epidemiology, adults.

\section{REFERENCIAS BIBLIOGRÁFICAS}

Abdul Rahman, N.; Ramli, R.; Abdul Rahman, R.; Hussaini, H. M. \& Abdul Hamid, A. L. Facial trauma in geriatric patients in a selected Malaysian hospital. Geriatr. Gerontol. Int., 10(1):64-9, 2010.

Acevedo, J. P.; Cooper M., H.; Tirreau T., V. \& Núñez, F. Descripción del traumatismo dentoalveolar en pacientes adultos. Rev. Dent. Chile, 97(1):8-13, 2006.

Andreasen, J. O.; Lauridsen, E. \& Christensen, S. S. Development of an interactive dental trauma guide. Pediatr. Dent., 31(2):133-6, 2009.

Caldas, A. F. Jr. \& Burgos, M. E. A retrospective study of traumatic dental injuries in a Brazilian dental trauma clinic. Dent. Traumatol., 17(6):250-3, 2001.

Comisión Nacional de Seguridad de Tránsito (CONASET). Evolución de Siniestros de Tránsito Chile 1972-2012. Santiago de Chile, Ministerio de Transporte y Telecomunicaciones, Gobierno de Chile, 2012.

Glendor, U. Epidemiology of traumatic dental injuries--a 12 year review of the literature. Dent. Traumatol., 24(6):60311, 2008.

Glendor, U. Aetiology and risk factors related to traumatic dental injuries--a review of the literature. Dent. Traumatol., 25(1):19-31, 2009.

Goldstein, E.; Universidad de Chile \& Centro de Estudios en Seguridad Ciudadana. Los robos con violencia en el Gran
Santiago: magnitudes y características. Santiago de Chile, Universidad de Chile, Instituto de Asuntos Públicos, Departamento de Políticas Públicas, 2003.

Guedes, O. A.; de Alencar, A. H.; Lopes, L. G.; Pécora, J. D. \& Estrela, C. A retrospective study of traumatic dental injuries in a Brazilian dental urgency service. Braz. Dent. J., 21(2):153-7, 2010

Laverick, S.; Patel, N. \& Jones, D. C. Maxillofacial trauma and the role of alcohol. Br. J. Oral Maxillofac. Surg., 46(7):542-6, 2008.

Locker, D. Self-reported dental and oral injuries in a population of adults aged 18-50 years. Dent. Traumatol., 23(5):291-6, 2007.

Ministerio de Salud (MINSAL). Guía práctica clínica de Urgencias Odontológicas. Santiago de Chile, Ministerio de Salud, Gobierno de Chile, 2011.

Onetto, J. E.; Flores, M. T. \& Garbarino, M. L. Dental trauma in children and adolescents in Valparaiso, Chile. Endod. Dent. Traumatol., 10(5):223-7, 1994.

Ramli, R.; Rahman, N. A.; Rahman, R. A.; Hussaini, H. M. \& Hamid, A. L. A retrospective study of oral and maxillofacial injuries in Seremban Hospital, Malaysia. Dent. Traumatol., 27(2):122-6, 2011.

Santos, S. E.; Marchiori, E. C.; Soares, A. J.; Asprino, L.; de Souza Filho, F. J.; de Moraes, M. \& Moreira, R. W. A 9year retrospective study of dental trauma in Piracicaba and neighboring regions in the State of São Paulo, Brazil. J. Oral Maxillofac. Surg., 68(8):1826-32, 2010. 
VELÁSQUEZ, F.; MANCILLA, C.; NIÑO, A. Y.; TIRREAU, V.; CORTÉS-ARAYA, J.; ROJAS, M. C.; ESCOBAR, E.; REYES-COURT, D.; CALLEJA, S.; ULLOA-MARÍN, C. \& SUNG-HSIEH, H. H. Patrones epidemiológicos del trauma dentoalveolar (Patología GES) en pacientes adultos atendidos en un Centro de Trauma de Chile durante 2 períodos. Int. J. Odontostomat, 8(2):191-199, 2014

SENDA, Observatorio Chileno de Drogas. Las principales estadísticas sobre consumo de drogas y alcohol de la serie de Estudios de Población General de SENDA, de 1994 a 2012. 2012. Disponible en: http:// www.senda.gob.cl/observatorio/estadisticas/poblaciongeneral/

Shaikh, Z. S. \& Worrall, S. F. Epidemiology of facial trauma in a sample of patients aged 1-18 years. Injury, 33(8):66971, 2002.

Sistema Integrado Estadístico de Carabineros de Chile (SIEC). Anuario estadístico de los Accidentes de Tránsito ocurridos en Chile, durante el año 2012. Santiago de Chile, Carabineros de Chile, 2012. p.19.

Solimano, G. Consumo de alcohol y sus efectos sobre la salud. Rev. Chil. Salud Pública, 10(3):177-81, 2006.

Urzúa, I.; Huberman, J.; Delgado, I.; Pacheco, A. \& Retamal, $R$. Prevalencia de caries y pérdida de dientes de una población adulta chilena nacida en el siglo XIX. Rev. Clin. Periodoncia Implantol. Rehabil. Oral, 2(3):175-8, 2009.
Correspondence:

Hsiao Hsin Sung Hsieh

Oral and Maxillofacial Surgery Department

University of Michigan

Ann Arbor

USA

Phone: (1) 734-9454695

Fax: (1) 734-6151415.

Email: sunghsie@med.umich.edu

Received: 27-04-2014

Accepted: 01-08-2014 